\begin{tabular}{|c|c|c|}
\hline $\begin{array}{l}\text { ITC 3/47 } \\
\text { Journal of Information Technology }\end{array}$ & \multicolumn{2}{|c|}{$\begin{array}{c}\text { Soft Variable Structure Control of Linear Systems via } \\
\text { Desired Pole Paths }\end{array}$} \\
\hline $\begin{array}{l}\text { Vol. } 47 / \text { No. } 3 / 2018 \\
\text { pp. } 447-456\end{array}$ & Received 2017/08/10 & Accepted after revision 2018/07/26 \\
\hline $\begin{array}{l}\text { DOI 10.5755/j01.itc.47.3.18805 } \\
\text { ๑ Kaunas University of Technology }\end{array}$ & \multicolumn{2}{|c|}{ ress ${ }^{2}$ http://dx.doi.org/10.5755/j01.itc.47.3.18805 } \\
\hline
\end{tabular}

\title{
Soft Variable Structure Control of Linear Systems via Desired Pole Paths
}

\section{Kaheni, M. Hadad Zarif, A. Akbarzadeh Kalat}

Faculty of Electrical Engineering and Robotics, Shahrood University of Technology, Shahrood, Iran; phone: +98 233239 2204; fax: +98 233239 2209; e-mails: mojtaba.kaheni@shahroodut.ac.ir, mhzarif@shahroodut.ac.ir, akbarzadeh@shahroodut.ac.ir

\section{Sami Fadali}

Department of Electrical and Biomedical Engineering, Faculty of Engineering, the University of Nevada, Reno, USA; phone: +1775 784 6951; fax: +1 775 7846627; e-mail: fadali@unr.edu

Corresponding author: mojtaba.kaheni@shahroodut.ac.ir

In this article, a novel method of soft variable structure control of linear systems, desired pole paths, is proposed. The proposed method is helpful to reach a fast response when a continuous control signal and satisfaction of some constraints are desired. The method selects a desired path for closed loop poles instead of the exact location of poles, then the pole placement in this path is determined by solving an optimization problem subject to a control signal constraint leading to a suboptimal control structure. The stability of the proposed method is provided based on the multivariable circle criterion and the Kalman-Yakubovich-Popov lemma. A design parameter is also introduced in this paper which can adjust a tradeoff between speed of response and smoothness of the control signal. Simulation of a satellite model shows an improvement in shortening the settling time and softening the control signal compared to published soft variable structure control schemes.

KEYWORDS: Circle criterion, Desired pole paths, Linear control, Nonlinear control, Variable structure control.

\section{Introduction}

Achieving a fast response while shortening the settling time is one of the main objectives in an industrial control process. Time optimal control which achieves the fastest settling time can be implemented via bangbang control [12]. Although the fast response of bangbang control is desirable, discontinuity and chatter- 
ing of the control signal reduce actuator service life and increase maintenance cost. Other drawbacks are the high control effort and sensitivity to uncertainties and disturbances. Therefore, researchers have proposed methods which have settling time close to time optimal control, a continuous control signal, and reduced control effort [1, 4, 9-10, 14-16, 23].

Variable structure control (VSC) is a strategy in which the control law varies as a function of the state and switches between $k$ pre-designed controllers [1]. The discontinuous function which selects the desired controller is called the selection function. Sliding mode control [19, 22], high order sliding mode control [4, 9], supervisory control [2-3], hybrid control [6-7], etc., are some examples of VSC. In VSC, sudden changes in the control law lead to high frequency switching, which is not desirable in industrial applications. If the number of pre-designed controllers goes to infinity and the selection function becomes continuous, this disadvantage is eliminated and the method is called soft variable structure control (SVSC). SVSCs can be utilized instead of bang-bang control when both a short settling time and continuous control signal are desired. The main methods that have been proposed to date in the literature for SVSC, which improve regulation rate, are divided into three main categories:

1 Soft variable structure control employing an implicit Lyapunov function [1]

2 Dynamical soft variable structure control $[1,10,15$ $16,23]$

3 Soft variable structure control with variable saturation $[1,14]$

Huge calculation is an obstacle in online implementation of SVSC employing an implicit Lyapunov function. This control method is also too conservative and rarely allows the capacity of the control signal to be fully exploited to decrease settling time. Dynamical SVSC methods have become more popular recently and their applications in singular [15-16, 23] and fractional order systems [10] have been reported in the literature. Although their structure is more suited to continuous time models, the response of the system is highly dependent on a designer-defined control vector. Because no relationship between the control vector and performance is available, good performance is highly dependent on the choice of control vector. SVSC with variable saturation, which is recently de- veloped with S class functions [14], has strict conditions to satisfy the input restriction that may prevent efficient utilization of the control signal. In this article, a novel method is proposed to improve SVSC and resolve the aforementioned obstacles.

The remainder of this paper is organized as follows. After this introduction in the second section, the problem of restricted control signal systems is described. In the third section, a feedback approach algorithm is proposed. The desired poles path approach is described in Section 4. Simulation results are given in Section 5 and conclusions in Section 6.

\section{Problem Description}

Consider a single input, single output (SISO) controllable linear system in the state space with differential equation

$$
\dot{\mathbf{x}}=A \mathbf{x}+\mathbf{b} u,
$$

where $\mathbf{x} \in \mathcal{R}^{n}, u \in \mathcal{R}$. The control input signal is restricted as

$$
|u| \leq u_{0} .
$$

Without loss of generality we assume that (1) is in standard controllable form or can be transformed into it. Thus, the state matrix $A$ and the input matrix b are in the form

$$
A=\left[\begin{array}{ccccc}
0 & 1 & 0 & \cdots & 0 \\
0 & 0 & 1 & \cdots & 0 \\
\vdots & \vdots & \vdots & \ddots & \vdots \\
0 & 0 & 0 & \cdots & 1 \\
-a_{0} & -a_{1} & -a_{2} & \cdots & -a_{n-1}
\end{array}\right], \mathbf{b}=\left[\begin{array}{c}
0 \\
0 \\
\vdots \\
0 \\
1
\end{array}\right]
$$

We require a continuous, fast and stable controller that satisfies the control signal constraint (2), with feasible initial state $\mathbf{x}_{0} \in X_{0} \subset \mathcal{R}^{n}$. Clearly, the requirement of a continuous control signal excludes bangbang control in this problem.

\section{State Feedback Control Approach}

One can choose a state feedback control strategy and endeavor to place the system poles where both stability and the input constraint are met while minimizing 
settling time. Let us recall some preliminaries.

Theorem 1. Lyapunov Stability [11]

Consider a system with the differential equation, $\dot{\mathbf{x}}=$ $f(x)$, where $f(x)$ is a continuous function with equilibrium point $\mathbf{x}=0$. If there exists a function $V(\mathbf{x})$ with continuous partial derivatives such that:

$V(0)=0$

$V(\mathbf{x})>0, \quad \mathbf{x} \neq 0$

$\dot{V}(\mathbf{x})<0, \quad \mathbf{x} \neq 0$,

then the equilibrium point, $\mathbf{x}=0$, will be asymptotically stable and $V(\mathbf{x})$ will be called a Lyapunov function. In linear systems as (1), the stability theorem is equivalent to finding a positive definite symmetric solution $P$ to the Lyapunov equation

$$
A^{T} P+P A=-Q
$$

for an arbitrary positive definite matrix, $Q$. The Lyapunov equation is derived using the quadratic Lyapunov functionm $V(\mathbf{x})=\mathbf{x}^{T} P \mathbf{x}$.

Definition 1. Lyapunov Region [1]

If there exists a function $V(\mathbf{x})$ that satisfies the conditions of Theorem 1 for a system $\dot{\mathbf{x}}=f(x)$ and $G=$ $\{\mathbf{x} \mid V(\mathbf{x})<c\}$ is bounded, then due to negativity of $\dot{V}(\mathbf{x})$, $G$ is an invariant set and is known as a Lyapunov region.

Clearly, placing the closed-loop poles in locations with smaller real values speeds up the response. In this section, we introduce a feedback gain vector function, $\mathbf{k}^{T}(v)$, that places the closed-loop poles on the pole ray depicted in Figure 1 as a function of $v$. We define $\mathbf{k}^{T}(v)$ such that

$v_{1}<v_{2} \Rightarrow$
$\operatorname{Re}\left(\operatorname{eig}\left(A_{c}\left(v_{1}\right)\right)\right)<\operatorname{Re}\left(\operatorname{eig}\left(A_{c}\left(v_{2}\right)\right)\right)$

where $A_{c}(v)=\left(A-\mathbf{b k}^{T}(v)\right)$.

The following procedure determines the optimum feedback gain that provides a fixed structure, fast and stable control, and satisfies the input signal constraint (2).

Procedure 1: Initialize with a flag value flag $=0$.

Step 1: Select an appropriate pole ray considering the desired or allowed overshoot as depicted in Figure 1.

Step 2: Select a suitable set of poles on the pole paths

\section{Figure 1}

Pole paths

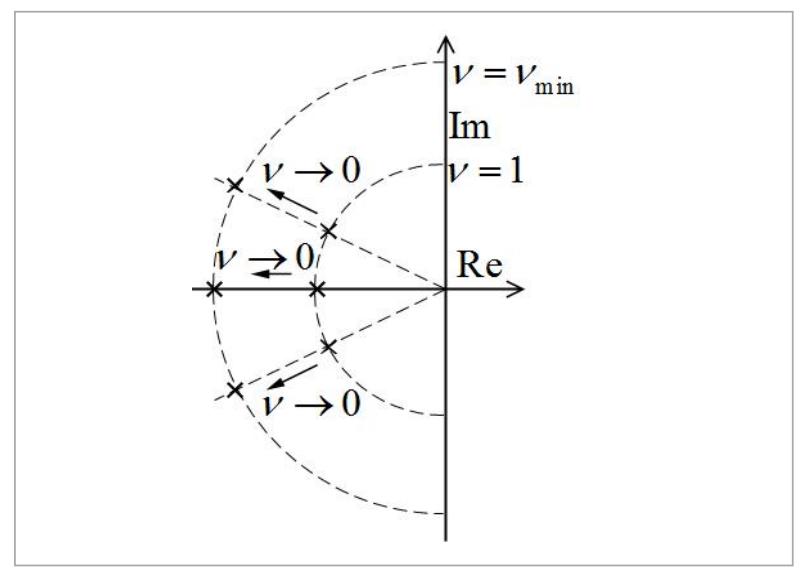

and consider $v=1$ for this set.

Step 3: Select suitable positive variation steps, $s_{1}$ and $s_{2}$ with $s_{2} \ll s_{1}<1$. For example, $s_{1}=0.2$ and $s_{2}=0.02$ would be appropriate.

Step 4: Find the feedback gain $\mathbf{k}^{T}(v)$ that leads to a closed loop system with the selected poles. If the state equation of the desired closed loop system is

$$
\dot{\mathbf{x}}=\left(A-\mathbf{b k}^{T}(v)\right) \mathbf{x}=A_{c}(v) \mathbf{x},
$$

then the control vector $\mathbf{k}(v)$ will be evaluated as follows:

$$
\mathbf{k}(v)=\left[\begin{array}{c}
\hat{a}_{0} v^{-n}-a_{0} \\
\hat{a}_{1} v^{-(n-1)}-a_{1} \\
\vdots \\
\hat{a}_{n-1} v^{-1}-a_{n-1}
\end{array}\right],
$$

where $\hat{a}_{j}, j=0,1, \ldots, n-1$, are the coefficients of the characteristic polynomial of $A_{c}(v=1)$.

Step 5: Solve the Lyapunov equation (4) of the closed loop system for the positive definite matrix, $P$, to obtain the Lyapunov function $V(\mathbf{x})=\mathbf{x}^{T} P \mathbf{x}$.

Step 6: Using the Lyapunov function, find a Lyapunov region $G=\{\mathbf{x} \mid V(\mathbf{x})<c\}$ in which the input constraint is satisfied. To fully exploit the control signal, the hyperplanes $\pm \mathbf{k}^{T} \mathbf{X}=u_{0}$ must be tangent to the Lyapunov region. To determine an appropriate $c$ value in the Lyapunov region formula, solve the optimization problem 


$$
\begin{gathered}
\max \mathbf{x}^{T} P \mathbf{x} \quad \text { s.t. } \\
\left|\mathbf{k}^{T} \mathbf{x}\right|<u_{0} .
\end{gathered}
$$

The solution of ( 7$)$ is $c=u_{0}^{2} / \mathbf{k}^{T} P^{-1} \mathbf{k}$.

Step 7: If $X_{0} \not \subset G$, change $v$ to $v=v+s_{2}$, set flag $=1$, and go to Step 4.

Step 8: If $X_{0} \subset G$ and flag $=0$, change the parameter $v$ to $v=v-s_{1}$ and go to Step 4. If $X_{0} \subset G$ and flag $=1$, stop.

The flowchart of Procedure 1 is depicted in Figure 2.

Figure 2

Flowchart of Procedure 1

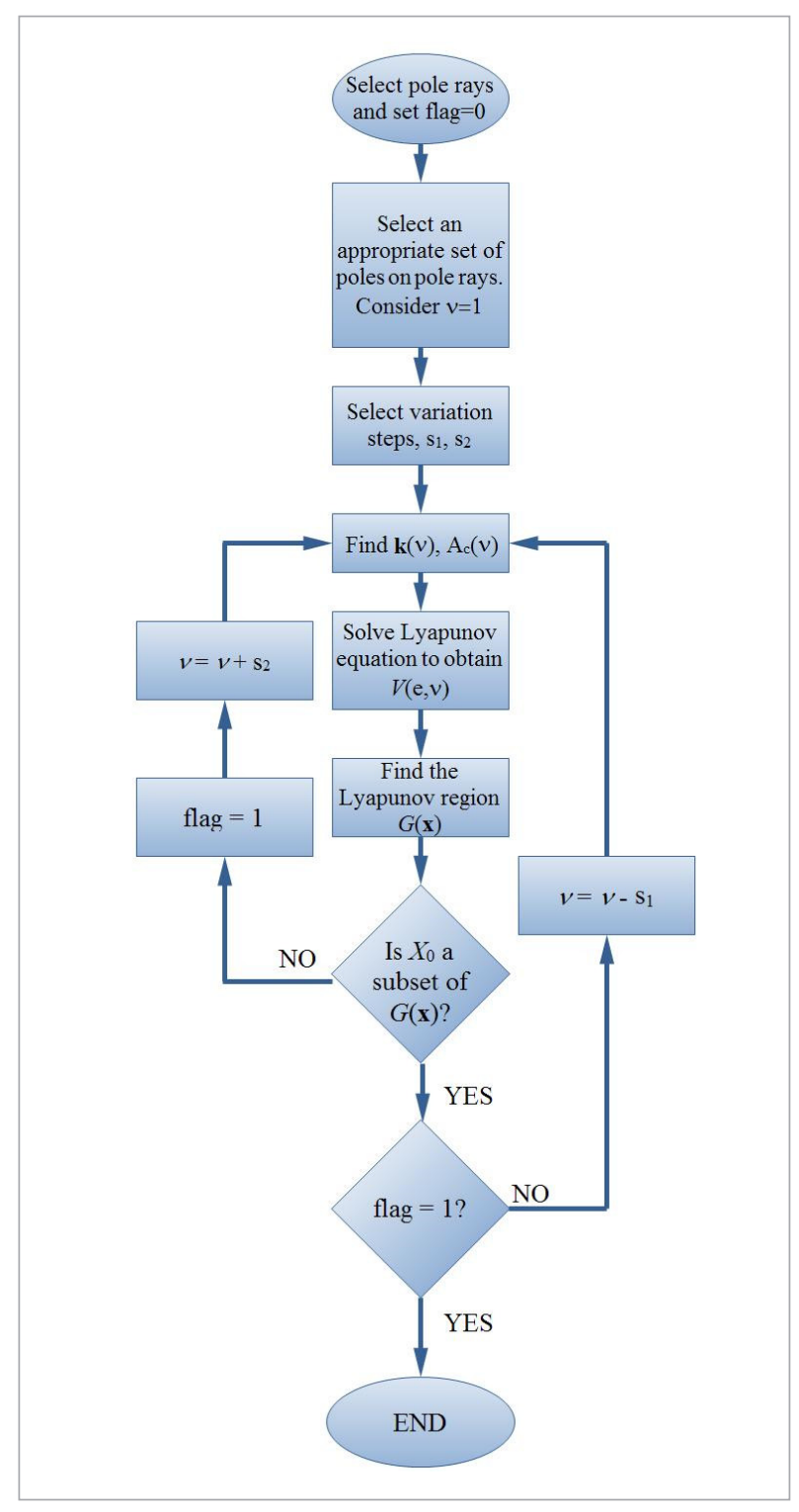

Remark 1: Procedure 1 determines the smallest $v$ that yields a Lyapunov region that contains $X_{0}$. The algorithm is initialized with a $v$ value that corresponds to a Lyapunov region that does not contain $X_{0}$. It then progressively adds the variation step, $s_{2}$, and checks $X_{0} \subset G$ until it reaches an optimum $v$ value. The flag prameter is defined to make the initialized value of $v$ small enough for $X_{0} \not \subset G$.

Remark 2: If the feasible initial condition set $X_{0}$ is a subset of $G$, then this state feedback control vector will satisfy the input constraint. Since $G$ is an invariant set and $X_{0} \subset G$, the state trajectory cannot leave the Lyapunov region of Step 4 and the input signal always satisfies (2). Figure 3, illustrates this situation. Procedure 1 tries to find the smallest $v$ that leads to a Lyapunov region $G$, where $X_{0} \subset G$.

\section{Figure 3}

Phase plane diagram of a second order system when $X_{0} \subset G$

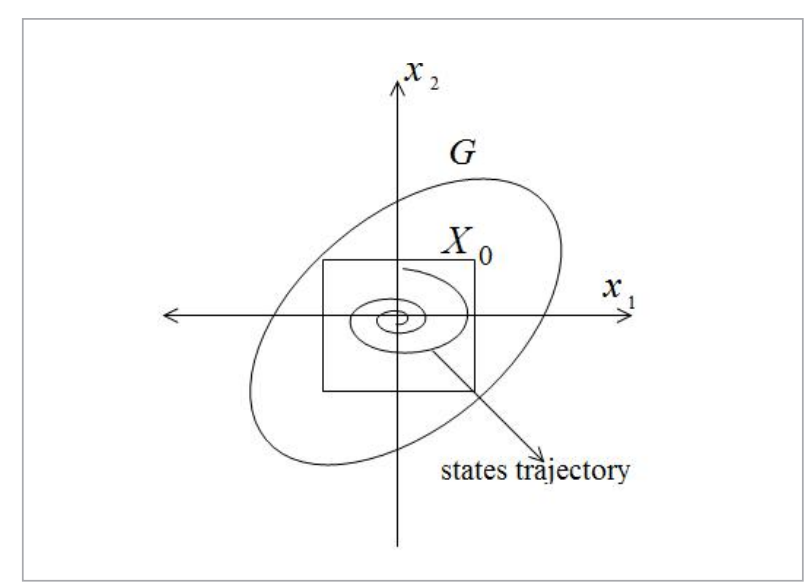

Remark 3: The method discussed in this section is a fixed structure control approach. Once the best pole placement, which satisfies the constraints, is found offline, the fixed respective control law will be applied to the plant.

\section{SVSC via Desired Pole Path Approach}

The method proposed in Section 3, is a good fix structure solution in designing a rapid controller with continuous control signal. But during the regulation period the amplitude of states will decrease, therefore it 
is possible to implement a faster feedback gain on the pole path during regulation period while considering input signal constraints. A novel algorithm, which is named SVSC via desired pole paths (DPP), is proposed in this section. First, a desired path of closed loop poles must be determined with a well-defined feedback gain as a function of a parameter $v$ as shown in Section 3. If we choose a ray-like path as in Figure 1 , the speed of response of the closed loop system will increase by decreasing $v$. Therefore, to obtain the fastest system response, we must minimize $v$ subject to the control signal constraints and the online state vector. The implementation steps of SVSC via desired poles path are as follows:

\section{Procedure 2}

Step 1: Run Procedure 1.

Step 2: Set $v=1$ for the result of Step 1.

Step 3: Get the online state vector, $x$.

Step 4: Solve the optimization problem

$\min v \quad$ s.t.
$v_{\min } \leq v \leq 1$
$\left|\mathbf{k}^{T}(v) \mathbf{x}\right|-u_{0} \leq 0$

where $v_{\text {min }}>0$.

Step 5: Apply $-\mathbf{k}^{T}(v) \mathbf{x}$ to the system.

The best feedback controller in the selected path, which is rapid and satisfies the control signal constraints, is $-\mathbf{k}^{T}(v) \mathbf{x}$, where $v$ is the solution of the optimization problem of Step 4 . Figures 4 and 5 depict the flowchart and scheme of DPP method, respectively.

Remark 4: According to (6) and (8), a polynomial programming problem must be solved in Step 4. The solution can be obtained by heuristic and Artificial Intelligence (AI) methods like Genetic Algorithm (GA) [13], Tabu search [18], Bees Algorithm [5] and Particle Swarm Optimization (PSO) [8], but the recursive nature of these methods is not acceptable in online implementation. One can discuss about the convexity of this problem and use classical optimization methods to solve it. But due to simplicity of this problem, finding the feasible space of the variable $v$ according to constraints is suggested. The feasible space of $v$ is the intersection of the range $\left[v_{\min }, 1\right]$ and the region bounded by the two polynomials of $v$ corresponding to the second constraint. The latter can be found using sign determination of polynomials. Next, the optimal value of the objective function can be easily determined in the feasible space.

\section{Figure 4}

Flowchart of Procedure 2

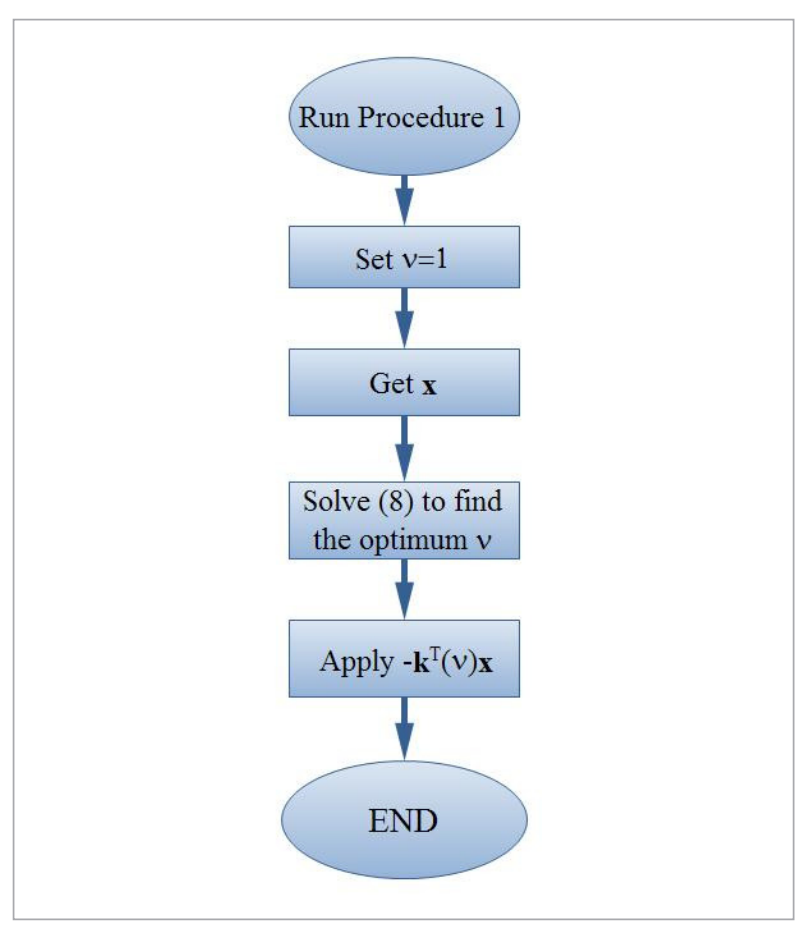

Figure 5

Desired pole paths block diagram

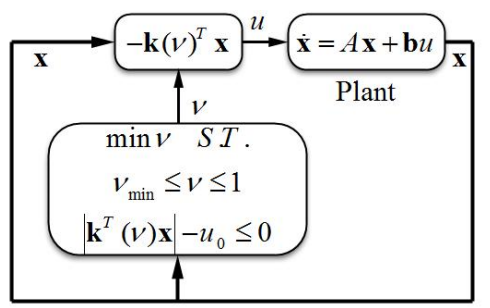

Remark 5: The appropriate selection of $v_{\min }$ in (8) is dependent to the stability of the closed loop system and will be discussed later in this section.

\section{Stability analysis of desired pole path SVSC}

By implementing this control scheme, the time invariant system becomes a continuously switching system. Maintaining the stability of the system be- 
comes more challenging and must be verified.

From (3), (5) and (6) we have

$$
\begin{aligned}
& \mathrm{A}_{c}(v)= \\
& {\left[\begin{array}{ccccc}
0 & 1 & 0 & \cdots & 0 \\
0 & 0 & 1 & \cdots & 0 \\
\vdots & \vdots & \vdots & \cdots & \vdots \\
0 & 0 & 0 & \cdots & 1 \\
-\hat{a}_{0} v^{-n} & -\hat{a}_{1} v^{-(n-1)} & -\hat{a}_{2} v^{-(n-2)} & \cdots & -\hat{a}_{n-1} v^{-1}
\end{array}\right]}
\end{aligned}
$$

We rewrite (9) as

$$
\mathrm{A}(v)=\mathrm{A}_{c}(1)-\mathrm{RM}(v),
$$

where $\mathrm{A}(1)=\left.\mathrm{A}(v)\right|_{v=1}, \mathrm{R}, \mathrm{M}(v) \in \mathcal{R}^{n \times n}$ and

$$
\mathrm{R}=\left[\begin{array}{c}
0_{(\mathrm{n}-1) \times \mathrm{n}} \\
1_{(1 \times n)}
\end{array}\right],
$$

$$
\mathrm{M}(v)=\operatorname{diag}\left\{\hat{a}_{0} v^{-n}-\hat{a}_{0}, \cdots, \hat{a}_{n-1} v^{-1}-\hat{a}_{n-1}\right\} .
$$

The representation (10) is in the form of the closedloop state matrix of a linear system with state feedback. Figure 6 depicts this configuration.

\section{Figure 6}

Feedback representation of desired pole path approach

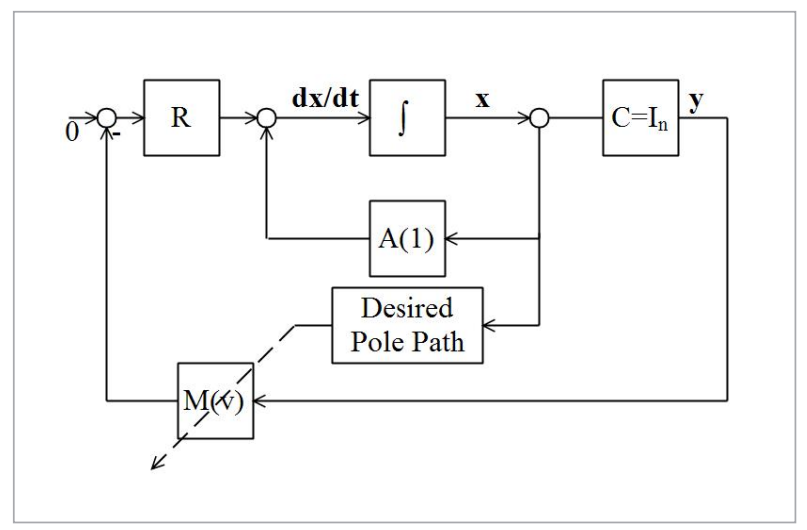

However, because of switching the value of the parameter $\mathrm{v}$ changes abruptly, the overall system is nonlinear. We investigate the bound $v=\left[v_{\text {min }}, 1\right]$, which guarantees the stability of (9) using the multivariable circle criterion.

Theorem 2. (Multivariable Circle Criterion) [11]
Consider a controllable and observable MIMO feedback system

$$
\begin{aligned}
& \dot{\mathbf{x}}=A \mathbf{x}+B \mathbf{u} \\
& \mathbf{y}=C \mathbf{x} \\
& \mathbf{u}=-\varphi(t, \mathbf{y}),
\end{aligned}
$$

where $\mathrm{x} \in \mathcal{R}^{n}, \mathbf{u}, \mathbf{y} \in \mathcal{R}^{p}, A$ is Hurwitz and $\varphi:[0, \infty) \times$ $\mathcal{R}^{p} \rightarrow \mathcal{R}^{p}$ is a memoryless, possibly time varying nonlinearity which is piecewise continuous in $t$ and locally Lipschitz in $\mathbf{y}$. If $\varphi(t, y)$ is sector bounded by 0 and $K_{\max }$ y globally

$$
\begin{aligned}
& \varphi^{\mathrm{T}}(t, \mathbf{y})\left[\varphi(t, \mathbf{y})-K_{\max } \mathbf{y}\right] \leq 0 \\
& \forall \mathrm{t} \geq 0, \quad \forall \mathbf{y} \subset \mathcal{R}^{P}
\end{aligned}
$$

and $Z(s)=I_{n}+K_{\max } C\left(s I_{n}-\mathrm{A}\right)^{-1} \mathrm{~B}$ is strictly positive real (SPR), then the feedback system is absolutely stable.

To derive our result, we need the following lemma.

Lemma 1. (Kalman-Yakubovich-Popov) [11]

Let $Z(s)=\mathrm{C}\left(s I_{n}-\mathrm{A}\right)^{-1} \mathrm{~B}+\mathrm{D}$ be a $p \times p$ transfer function matrix, where A is Hurwitz, (A, B) is controllable, and (A, $\mathrm{C})$ is observable. Then, $Z(s)$ is strictly positive real if and only if there exist a positive definite symmetric matrix $P$, matrices $W$ and $L$, and a positive constant $\varepsilon$ such that

$$
\begin{aligned}
& \mathrm{PA}+\mathrm{A}^{\mathrm{T}} \mathrm{P}=-\mathrm{L}^{\mathrm{T}} \mathrm{L}-\varepsilon \mathrm{P} \\
& \mathrm{PB}=\mathrm{C}^{\mathrm{T}}-\mathrm{L}^{\mathrm{T}} \mathrm{W} \\
& \mathrm{W}^{\mathrm{T}} \mathrm{W}=\mathrm{D}+\mathrm{D}^{\mathrm{T}} .
\end{aligned}
$$

\section{Theorem 3. (Stability of DPP control)}

The desired pole path control of (1) is stable if there exist a positive semidefinite symmetric matrix $P$, matrix $L$ and a positive constant $\varepsilon$ such that

$$
\begin{aligned}
& \mathrm{PA}_{c}(1)+\mathrm{A}_{c}(1)^{T} \mathrm{P}=-\mathrm{L}^{\mathrm{T}} \mathrm{L}-\varepsilon \mathrm{P} \\
& \mathrm{PR}=\mathrm{M}\left(v_{\text {min }}\right)^{\mathrm{T}}-\sqrt{2} \mathrm{~L}^{\mathrm{T}} .
\end{aligned}
$$

Proof: We first show that the assumptions of Theorem 1 hold for our system. Clearly, the pair $\left(A_{c}(1), R\right)$ is in controllable form and is therefore controllable. The pair $\left(\mathrm{A}_{c}(1), I_{n}\right)$ is observable and since all the eigenvalues are located in the left hand plane by Procedure 1, $\mathrm{A}_{c}(1)$ is Hurwitz for $v=1$. We need to check the following two properties:

$1 \mathrm{M}(v)$ is piece-wise continuous.

From (8), since $\mathrm{v}=1$ always satisfies the constraints, the optimal solution of (8) is a root of the polynomial 
$\left(\mathbf{k}^{T}(v) \mathbf{x}\right)^{2}=u_{0}^{2}$ or $v_{\text {min }}$. Since the coefficients of $\left(\mathbf{k}^{T}(v) \mathbf{x}\right)^{2}$ are continuous functions of the state, they vary continuously with the state, as do the roots of the polynomials [17]. Therefore, $\mathrm{M}(v)$ is always piecewise continuous.

$2 \mathbf{y}^{\mathrm{T}} \mathrm{M}(v)^{\mathrm{T}}\left[\mathrm{M}(v) \mathbf{y}-\mathrm{M}\left(v_{\min }\right) \mathbf{y}\right] \leq \mathbf{0}$.

From the definition of $\mathrm{M}(v)$, since $a_{i}>0$,for $i=1, . ., n-$ 1 and $0<v_{\text {min }}<v<1, \mathrm{M}(v)>0$ and $\forall v \in\left[v_{\text {min }}, 1\right), \mathrm{M}(\mathrm{v}) \leq$ $\mathrm{M}\left(v_{\text {min }}\right)$. Therefore

$$
\begin{aligned}
& \mathrm{M}(v)^{\mathrm{T}}\left[\mathrm{M}(v)-\mathrm{M}\left(v_{\text {min }}\right)\right] \leq \mathbf{0} \\
& \mathbf{y}^{\mathrm{T}} \mathrm{M}(v)^{\mathrm{T}}\left[\mathrm{M}(v)-\mathrm{M}\left(v_{\text {min }}\right)\right] \mathbf{y} \leq \mathbf{0} \\
& \mathbf{y}^{\mathrm{T}} \mathrm{M}(v)^{\mathrm{T}}\left[\mathrm{M}(v) \mathbf{y}-\mathrm{M}\left(v_{\text {min }}\right) \mathbf{y}\right] \leq \mathbf{0}
\end{aligned}
$$

Thus, our system satisfies all the assumptions of Theorem 2.

Next, we verify that $I_{n}+\mathrm{M}\left(v_{\text {min }}\right)\left(s I_{n}-A(1)\right)^{-1} R$ is positive real. Applying Lemma 1 with the matrix $D=I_{n}$, the matrix is positive real matrix if there exist a positive semidefinite symmetric matrix $\mathrm{P}$, matrix $\mathrm{L}$ and a positive constant $\varepsilon$ such that (16) is satisfied.

Theorem 3 shows that the stability of the switching system (5) is guaranteed if we can find a positive semidefinite symmetric matrix $\mathrm{P}$, a matrix $\mathrm{L}$, and a positive constant $\varepsilon$ that satisfy (16).

A smaller $v_{\text {min }}$ is equal to larger sector in (14) and if a particular choice fails to find an appropriate $\mathrm{P}$ in (16), a larger $v_{\min }$ should be used to prove the stability.

Remark 6: A necessary (but not sufficient) condition for (16) to have a solution is that the matrix product $\mathrm{A}(1) \mathrm{A}\left(v_{\text {min }}\right)$ does not have any negative real eigenvalues [20]. This eliminates inadmissible values of $v_{\min }$ without solving (12).

\section{Simulation Results}

Figure 7 shows the control system for a simple satellite model [21]. The satellite is a rotational unit inertia controlled by a pair of thrusters that provide the desired torque between a positive constant torque $U$ (positive firing) and a negative torque $-U$ (negative firing). The goal of the control system is to maintain the satellite antenna at a zero angle by firing the thrusters in the admissible bound. The mathematical model of this system is:

$$
\ddot{\theta}=u \text {. }
$$

Figure 7

Satellite control system

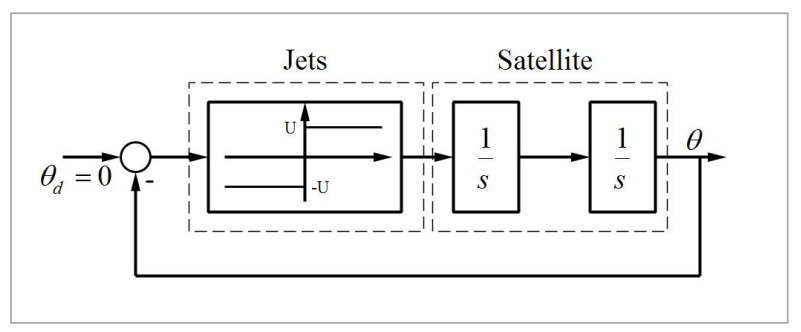

With $\theta=x_{1}$ and $\dot{\theta}=x_{2}$, the state equation of the system can be represented by

$$
\dot{\mathbf{x}}=\left[\begin{array}{ll}
0 & 1 \\
0 & 0
\end{array}\right] \mathbf{x}+\left[\begin{array}{l}
0 \\
1
\end{array}\right] u .
$$

Consider the feasible initial states $x_{1}(0)<1, x_{2}(0)<$ 1 , input signal constraint $|u| \leq 10$ and $y=x$. A poles path given by a radial line at $45^{\circ}$ angle from the negative real axis is desired. Running Procedure 1 leads to $\mathbf{k}=\left[\begin{array}{l}2 \\ 2\end{array}\right]$ as depicted in Figure 8.

\section{Figure 8}

Lyapunov region, constraints and feasible initial state region in phase plane

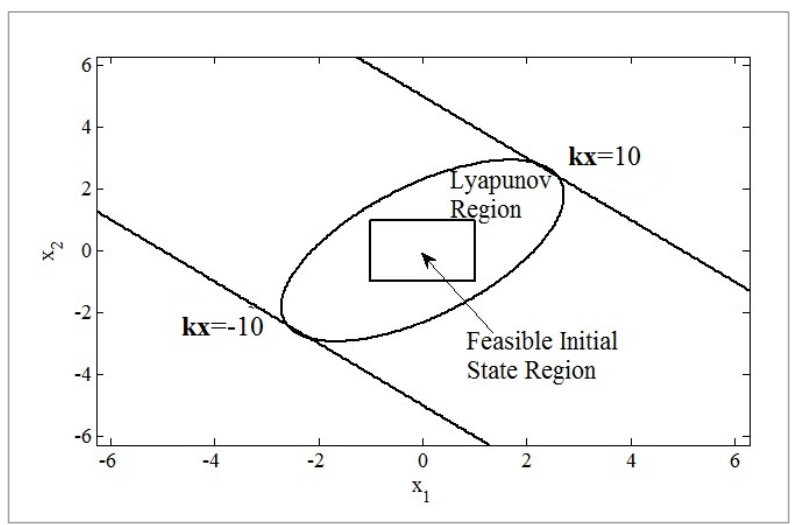

The closed loop dynamic equation considering Step 4 of Procedure 1 can be expressed by

$$
\dot{\mathbf{x}}=\left(A-\mathbf{b k}^{T}(v)\right) \mathbf{x}=\left[\begin{array}{cc}
0 & 1 \\
-2 v^{-2} & -2 v^{-1}
\end{array}\right] \mathbf{x} .
$$

It can be verified that if $v_{\min }=0.5$ is chosen, $\left[\begin{array}{cc}24 & 4 \\ 4 & 4\end{array}\right]$ will satisfy the conditions of Theorem 3 .

Figures 9 and 10 compare the response and the control signal of bang-bang control, state feedback con- 


\section{Figure 9}

Output responses, 1: bang-bang control, 2: state feedback control, 3: dynamical SVSC, 4: variable saturation SVSC and 5: the proposed DPP approach

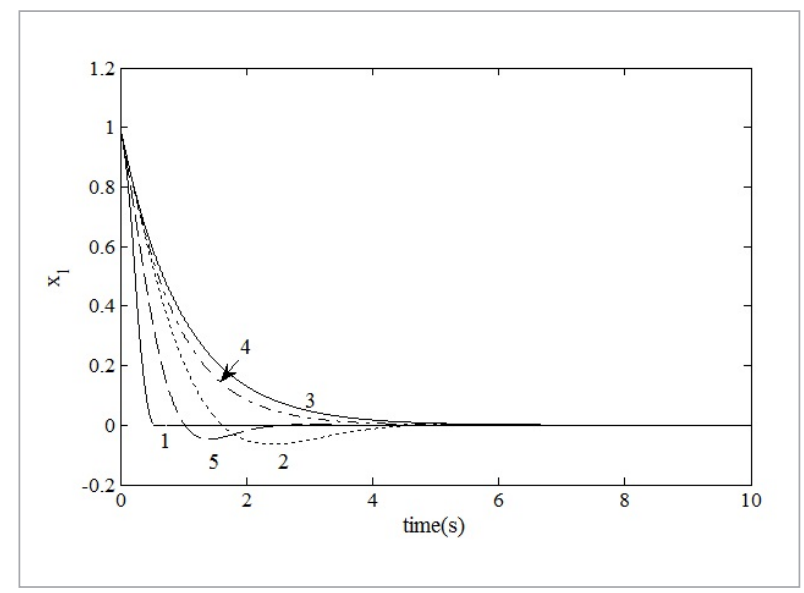

\section{Figure 10}

Control signals, 1: bang-bang control, 2: state feedback control, 3: dynamical SVSC, 4: variable saturation SVSC and 5: the proposed DPP approach

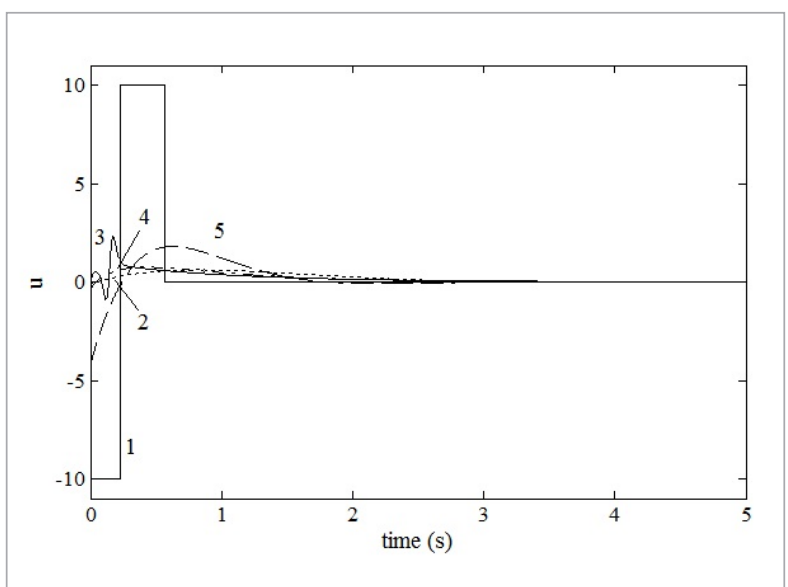

trol, dynamical SVSC, variable saturation SVSC and the proposed DPP approach to the $\mathbf{x}_{0}=[1,-0.95]^{\mathrm{T}}$ disturbance.

As shown in Figure 9, our proposed method, desired pole paths, has good performance compared to state feedback control, dynamical SVSC, and variable saturation SVSC. The output of our proposed method is close to the response of time optimal control while the control signal is continuous as shown in Figure 10. Figure 10 also shows that this good performance is achieved while the input signal constraints are satisfied.

Effect of $v_{\text {min }}$ : The response and the control signal of DPP controlled system with $v_{\text {min }}=0.3$ and $v_{\text {min }}=0.5$ are compared in Figures 11 and 12.

There is a tradeoff between speed of response and smoothness of the control signal. A larger $v_{\min }$ makes the control signal smoother but slows down the response while a smaller $v_{\min }$ speeds up the response with loss of control signal smoothness. The designer must choose a compromise value of $v_{\text {min }}$ depending on the specific design requirements.

Figure 11

Effect of $v_{\min }$ on the output responses

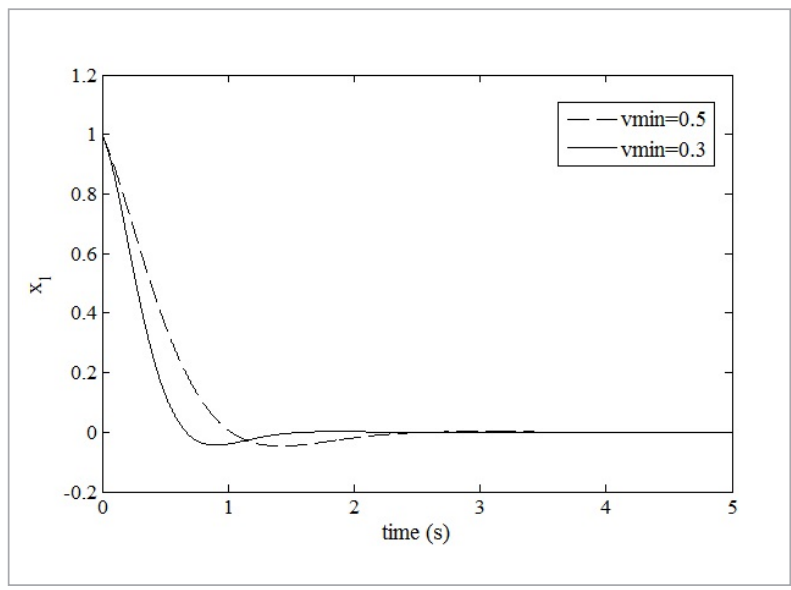

\section{Figure 12}

Effect of $v_{\min }$ on the control signals

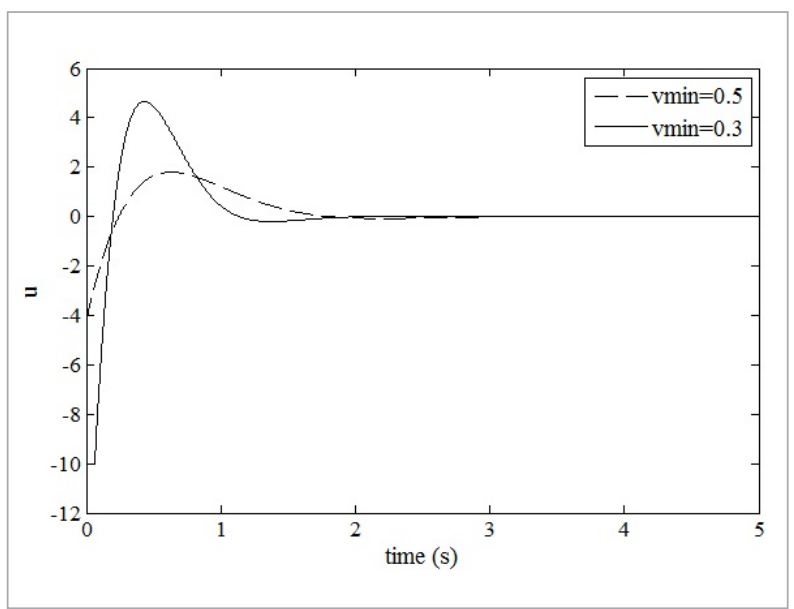




\section{Conclusion}

This paper proposes a new method to determine optimal locations for closed loop poles in selected pole paths to achieve a fast response and smooth control signal subject to constraints. By exploiting the fact that the norm of the state decays exponentially, the method increases the control gain progressively to achieve the desired time response. Our simulation results show that the method is superior to the previous SVSC approaches. Its response is close to bang-bang

\section{References}

1. Adamy, J., Flemming, A. Soft Variable-Structure Controls: A Survey. Automatica, 2004, 40(11), 1821-1844. https://doi.org/10.1016/j.automatica.2004.05.017

2. Cury, J. E. R., Hering de Queiroz, M., Bouzon, G., Teixeira, M. Supervisory Control of Discrete Event Systems with Distinguishers. Automatica, 2015, 56(1), 93-104. https://doi.org/10.1016/j.automatica.2015.03.025

3. Devaraj, R., Sarkar, A., Biswas, S. Comments on „Supervisory Control for Real-Time Scheduling of Periodic and Sporadic Tasks with Resource Constraints." Automatica, 2017, 82(1), 332-334. https://doi.org/10.1016/j. automatica.2017.04.007

4. Dinuzzo, F., Ferrara, A. Higher Order Sliding Mode Controllers with Optimal Reaching. IEEE Transactions on Automatic Control, 2009, 54(9), 2126-2136. https://doi. org/10.1109/TAC.2009.2026940

5. Gholipour, R., Khosravi, A., Mojallali, H. Multi-Objective Optimal Backstepping Controller Design for Chaos Control in a Rod-Type Plasma Torch System Using Bees Algorithm. Applied Mathematical Modelling, 2015, 39(15), 4432-4444. https://doi.org/10.1016/j. apm.2014.12.049

6. Goebel, R. Optimal Control for Pointwise Asymptotic Stability in a Hybrid Control System. Automatica, 2017, 81(1), 397-402. https://doi.org/10.1016/j.automatica.2017.04.021

7. Hu, B., Guan, Z., Jiang, X., Chi, M., Yu, L. On Consensus Performance of Nonlinear Multi-Agent Systems with Hybrid Control. Journal of the Franklin Institute, 2016, 353(13), 3133-3150. https://doi.org/10.1016/j.jfranklin.2016.05.010

8. Huang, H., Ma, G., Zhuang, Y., Lv, Y. Optimal Spacecraft Formation Reconfiguration with Collision Avoidance time-optimal control with a smooth control input thus eliminating the undesirable impact of discontinuous control on the system.

Future work will assess the robustness of this methodology and extend it to multivariable system, nonlinear systems, as well as singular and fractional order systems. Another research avenue for future investigation is the selection of pole paths and the introduction of a smoothness parameter in the design procedure.

Using Particle Swarm Optimization. Information Technology and Control, 2012, 41(2), 143-150. https://doi. org/10.5755/j01.itc.41.2.854

9. Incremona, G. P., Rubagotti, M., Ferrara, A. Sliding Mode Control of Constrained Nonlinear Systems. IEEE Transactions on Automatic Control, 2017, 62(6), 29652972. https://doi.org/10.1109/TAC.2016.2605043

10. Kamal, S., Bandyopadhyay, B. High Performance Regulator for Fractional Order Systems: A Soft Variable Structure Control Approach. Asian Journal of Control, 2015, 17(4), 1342-1346. https://doi.org/10.1002/asjc.1008

11. Khalil. H. K. Nonlinear Systems, Third Edition. Prentice-Hall, New Jersey, 2001.

12. Kirk, D. E. Optimal Control Theory: An Introduction. Dover Publications, New York, 2004.

13. Li, B., Du, H., Li, W. Optimal Distribution Control of Non-Linear Tire Force of Electric Vehicles With InWheel Motors. Asian Journal of Control, 2016, 18(1), 69-88. https://doi.org/10.1002/asjc.1145

14. Liu, Y., Gu, S., Kao, Y., Tang, S. Soft Variable Structure Controller Design for Constrained Systems Based on S-Class Functions. Neural Computing and Applications, 2015, 26(3), 705-711. https://doi.org/10.1007/ s00521-014-1748-0

15. Liu, Y., Kao, Y., Gu, S., Karimi, H. R. Soft Variable Structure Controller Design for Singular Systems. Journal of the Franklin Institute, 2015, 352(4), 1613-1626. https:// doi.org/10.1016/j.jfranklin.2015.01.030

16. Liu, Y., Zhang, C., Gao, C. Dynamic Soft Variable Structure Control of Singular Systems. Communications in Nonlinear Science and Numerical Simulation, 2012, 17(8). 3345-3352. https://doi.org/10.1016/j.cnsns.2011.11.039 
17. Marden, M. The Geometry of the Zeros of a Polynomial in a Complex Variable, First Edition. American Mathematical Society, 1949.

18. Misevičius, A., Smolinskas, J., Tomkevičius, A. Iterated Tabu Search for the Traveling Salesman Problem: New Results. Information Technology and Control, 2005, 34(4), 327-337.

19. Ngo, Q. H., Nguyen, N. P., Nguyen, C. N., Tran, T. H., Ha, Q. P. Fuzzy Sliding Mode Control of an Offshore Container Crane. Ocean Engineering, 2017, 140(1), 125-134. https://doi.org/10.1016/j.oceaneng.2017.05.019

20. Shorten, R. N., Narendra K. S. On Common Quadratic Lyapunov Functions for Pairs of Stable LTI Systems Whose System Matrices are in Companion Form. IEEE
Transactions on Automatic Control, 2003, 48(4), 618621. https://doi.org/10.1109/TAC.2003.809795

21. Slotine, J. E., Li, W. Applied Nonlinear Control. Prentice Hall, New Jersey, 1991.

22. Weng, Y. P., Gao, X. W. Data-Driven Sliding Mode Control of Unknown MIMO Nonlinear Discrete-Time Systems with Moving PID Sliding Surface. Journal of the Franklin Institute, 2017, 354(15), 6463-6502. https:// doi.org/10.1016/j.jfranklin.2017.07.022

23. Xu, R., Liu, Y., Gao, C., Wang, S. Soft Variable Structure Control with Differential Equation for Generalized Systems. The 26th Chinese Control and Decision Conference (2014 CCDC), Changsha, China, 2014, 530-535. https://doi.org/10.1109/CCDC.2014.6852205 